\title{
BMJ Open Developing a psychiatrist-patient relationship when both people are doctors: a qualitative study
}

\author{
Josephine Stanton, ${ }^{1}$ Patte Randal ${ }^{2}$
}

To cite: Stanton J, Randal P Developing a psychiatristpatient relationship when both people are doctors: a qualitative study. BMJ Open 2016;6:e010216.

doi:10.1136/bmjopen-2015010216

- Prepublication history for this paper is available online To view these files please visit the journal online (http://dx.doi.org/10.1136/ bmjopen-2015-010216).

Received 20 October 2015 Revised 29 March 2016 Accepted 28 April 2016

\section{ABSTRACT}

Objective: To better understand the complexities of developing an effective psychiatrist-patient relationship when both people involved are doctors.

Method: In-depth, semistructured interviews were conducted with 11 doctors with experiences as patients of psychiatrists (DPs) and eight psychiatrists with experience of treating doctors (TPS). A thematic analysis was undertaken.

Results: The medical culture of unrealistically high standards with limited room for vulnerability and fallibility, vigilance for judgment and valuing clinical over personal knowledge affected both people in the relationship. DPs struggled with the contradictions involved in entering the patient role but tried hard to be good patients. They wanted guidance but found it hard to accept and seldom communicated dissatisfaction or disagreement to their TPs. They described widely varying responses to diagnosis and treatment within the biomedical model. TPs described enjoyment and satisfaction and extreme challenge in engaging with TPs. Despite focusing on providing ordinary care they described providing extra care in many ways.

Conclusions: This study brings forward important issues when a psychiatrist is building a therapeutic relationship with another doctor. These are also likely to arise with other people and contribute to making truly patient-centred 'ordinary care' a hard ideal to fulfil. They include: (1) doctors' sense of ourselves as invincible, (2) TPs' sense of personal connection to, and identity with, DPs, (3) having extensive medical knowledge and (4) striving to be good patients. We need to make these issues explicit and enable the DP (or other patients) to tell their story and speak about their experience of the consultation so that any potential rupture in the therapeutic relationship can be addressed early.

\section{INTRODUCTION}

${ }^{1}$ Child and Family Unit, Auckland City Hospital, Auckland Mail Centre, Auckland, New Zealand ${ }^{2}$ Hamilton, New Zealand

Correspondence to Dr Josephine Stanton; josephines@adhb.govt.nz
Doctors are people too. Yet as a profession we have a tradition of holding superhuman expectations of ourselves and each other. ${ }^{1-4}$ Doctors in a range of contexts have been identified as holding a view that we, unlike our patients, are invulnerable to disease or just not permitted to be sick. ${ }^{2}{ }^{5-7}$ Doctors

\section{Strengths and limitations of this study}

- The major strength of the study is that it provides in-depth, experience-based data, where data are lacking: experiences of doctors engaging in mental healthcare with psychiatrists, including doctors with extreme mental health challenges.

- Another strength of the study is the different insider perspectives of the two authors including a doctor-patient perspective.

- Limitations of the study include a self-selection bias and a small sample size where doctors working in psychiatry are over-represented.

also fail to use usual traditional channels of help-seeking for healthcare. ${ }^{7}$ Mental health needs occur in doctors at rates at least equal to those in the general population and rates of completed suicide are higher. ${ }^{8}$ Despite this, levels of stigma attached to mental illness in doctors are still high in the medical profession. ${ }^{8}$ There appear to be particular barriers in seeking mental healthcare. ${ }^{5-12}$

The doctor-patient relationship has received increasing attention in recent decades. There is widespread commitment to patient-centred medicine and shared decision-making. ${ }^{13}$ The common elements of communication which contribute to building a therapeutic relationship include exploring patients' perspective, using open-ended questions and biomedically focused questions, showing empathy and joint strategy development. ${ }^{14} 15$ There are significant differences between and within individual patients, as to their preferences for a partnership model or more paternalistic approaches ${ }^{13} 16$ but the contribution of physician empathy to patient satisfaction is clear. ${ }^{17}$

The focus on patient-centred medicine and physician-patient communication is widespread in medicine generally, but attention to them within psychiatry has been limited. ${ }^{18}$ The quality of the therapeutic alliance has a consistent moderate relationship to psychotherapy outcomes. ${ }^{19} 20$ There is also 
an association between therapeutic relationship and outcomes across a range of areas of mental health, including severe mental illness. ${ }^{21}$ Psychiatric patients described the therapeutic, helping relationship as the most important aspect of care. ${ }^{22}$ Patients in assertive outreach teams described a partnership model of therapeutic relationship as important for engagement. ${ }^{23}$

Although there are no previous studies on the development of the psychiatrist-patient relationship when the patient is a doctor, studies on providing general healthcare to doctors and doctors' illness experience often include doctors with mental health issues. Primary care providers have reported that when treating doctors they experience an emotional connection, and an assumption of having their performance evaluated. ${ }^{10}$ The experience of providing care to another doctor was described as 'like sitting an exam'. ${ }^{24}$

Doctors in these studies experienced discomfort, shame and embarrassment with the patient role. ${ }^{7} 24$ They struggled with a number of issues; these included being unwell at all; imposing on another doctor's time and being judged for their level of concern about a trivial illness or for their own diagnosis and treatment. $^{2} 367112425$ They described being a doctor as an important aspect of their identity and as patients they were particularly aware of the potential risk to their ability to practice. ${ }^{3} 112627$ Maintaining control over their treatment was also an issue. ${ }^{24}{ }^{26}$ They tried to be good patients ${ }^{28}$ and described being watchful of the performance of their treating doctors. ${ }^{24} 26$ They wanted a doctor who showed competence and decisiveness, someone they could respect, preferably senior to them with limited emphasis on patient-centred qualities. ${ }^{24} 26$ They found it less easy to be open with a colleague and identified being treated differently because of being a doctor as unhelpful in some ways. ${ }^{24} 26{ }^{29}$ Doctors felt their lived experience as a patient improved their own professional practice. ${ }^{2425} 3031$

Doctors in these studies engaged in denial of their healthcare needs and chose doctors to consult who were less likely to challenge this denial. ${ }^{2}$ Doctors minimised attention to their own health needs and those of their general practitioner (GP) practice partners. ${ }^{11}$ Doctors' friends and colleagues had difficulty identifying and speaking with them about their mental health concerns. ${ }^{5}$ It may be that all the aforementioned issues are more apparent in the area of mental health. ${ }^{9-12}{ }^{32}$ In contrast, doctors and their families made good use of a

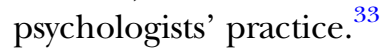

The aim of this paper is to present experiences of doctor-patients (DPs) and their treating-psychiatrists (TPs) in developing a therapeutic relationship.

\section{METHOD}

This was an exploratory qualitative study using in-depth interviews to obtain rich, descriptive accounts of the experiences of doctors, both as patients and as providers of treatment to doctors with mental health issues.

\section{Recruitment of participants}

Recruitment was challenging. Once we had gained ethical approval (AKY/04/12/344) we approached the Medical Council and a doctor who provided support to other doctors with mental health issues, for assistance. Both declined due to concerns regarding doctors' sensitivity about confidentiality. One of the researchers (PR) was the first participant. One participant was recruited via letters sent out to psychiatrists. Another offered to participate after hearing a presentation of some preliminary data. The remaining doctors were recruited via support networks (eg, a notice about the research was posted on an online support group website) or other participants. $^{5}$ Thirteen were approached and eight agreed to participate. Thus there were $11 \mathrm{DP}$ participants.

We approached 11 psychiatrists using purposive sampling to include a range of participants from different workplace settings and practice orientations (eg, private vs public practice, psychotherapy vs pharmacotherapy focus, roles with regard to Medical Council and other potential referrers of doctors). Eight agreed to be interviewed. All TPs were known to one or both of the researchers.

\section{Data collection}

Two interviews were conducted by JS alone, with the remainder undertaken by both of us together. Audio recordings were made of all interviews. Participants were encouraged to take the time they needed, and some DP interviews were completed over two meetings (11.5 hours in length). Interviews were held at a place of the participant's choice, and these included either their homes, a hotel or workplaces.

As part of the consent process, participants identified a support person and gave us permission to contact them if we had concerns for their well-being. Interviews started with open questions to encourage participants to speak freely, followed by more focused questioning to elicit greater detail. DPs were invited to speak about their experiences of identifying and living with mental health issues, accessing mental healthcare, and the development of their relationship with the treating psychiatrist; this was undertaken with a particular focus on the contribution of their professional role as a doctor. TPs were interviewed following the DPs. They were initially encouraged to talk about their experiences of, and responses to, engaging with DPs. Following this, they were specifically asked about the themes that emerged from the DP interviews (eg, role of professional knowledge, responses to receiving a diagnosis and medication, and reluctance to take time off work). As data analysis was ongoing during the data collection phase, the interview questions were modified and 
adapted throughout to take into account the emerging themes and ideas.

\section{Data analysis}

Interviews were transcribed, returned to participants for review and/or correction and then reviewed by JS, who removed any identifying data. Following this we developed a thematic framework. ${ }^{8}$ NVIVO $^{\mathrm{i}}$ (a data analysis software package) was used by both researchers to code the data independently, and results were compared and contrasted. Any differences identified (eg, exceptions for emerging themes) were discussed. This process was repeated over the period of the study, with the transcripts revisited and reviewed until agreement was reached. Draft analysis was emailed to participants for comment, ${ }^{34}$ with the analysis endorsed by all those who responded.

\section{Insider perspective}

As doctors working in psychiatry, both authors have insider perspectives (albeit different) with regard to the research topic. JS works in acute adolescent inpatient psychiatry (regularly using biological and psychological treatment approaches within the context of compulsory care) and PR has worked psychologically in a rehabilitation context with adults with severe and chronic psychiatric disorders.

PR has a more specific and significant insider perspective, in having had repeated experiences of psychosis and personal experience of engaging in care with psychiatrists about which she has written and spoken publicly. ${ }^{35-37}$ This was essential in the recruitment of participants and valuable in building trust. However, an insider perspective entails a risk that participants will assume a level of understanding, and that insider researchers may project their ideas onto participants (although outsider researchers are not immune to this ${ }^{38}$ ). There are also potential risks of researchers assuming understanding and limiting participants' disclosures to those consistent with the researcher's insider perspective. ${ }^{39}$ To address this risk, JS interviewed PR first and an analysis of her interview was undertaken before the other interviews, so that PR's insider perspective was explicitly available to both researchers. We could then look for similarities and differences with the other participants, and manage the influence of her perspective on the data collection and analysis processes. All interviews included a researcher (JS) without this insider perspective.

\section{RESULTS}

\section{Description of the sample}

Nineteen doctors took part in the study, including $11^{\mathrm{ii}}$ in the role of a DP and 8 TPs. There were 22 interviews in total $^{\mathrm{iii}}$. Within the DP category, five men and six women were interviewed, aged between 32 and 54 years. DPs identified themselves as being treated by their psychiatrists for the following diagnoses ${ }^{\text {iv }}$ : depression $(\mathrm{N}=8)$, psychosis $(\mathrm{N}=3)$, bipolar disorder $(\mathrm{N}=2)$, generalised anxiety disorder $(\mathrm{N}=1)$ and polysubstance dependence $(\mathrm{N}=1)$. At the time of initial identification of mental health needs, two had been working in psychiatry, six in general practice, three as hospital junior medical staff, one a student and one a specialist. Four changed career direction and were working in psychiatry at the time of the interviews. The length of time off work owing to mental illness varied from less than a month to 2 years.

Eight TPs were interviewed, including five men and three women, aged between 44 and 67 years. Participants' current employment at the time of the research included full time roles in either private practice or the public sector, or a mix of both. All engaged a range of strategies in their work, including some with a focus on psychotherapy.

\section{The culture doctors work in}

Both groups of participants spoke in some depth about the culture doctors work in. Medical culture, as they described it, was characterised by explicit and implicit demands for intellectual capacity, impeccable professionalism, extensive evidence-based knowledge, respect for seniority and authority and 'perfection' with regard to work performance. Several described poor self-worth despite high achievement beginning in childhood, and some recounted childhood patterns of denial of painful emotion. Rigorous expectations in medical school and the bullying culture of early work years were described as having strengthened patterns of self-dissatisfaction and having instilled a relentless striving for excellence. Participants reported limited room for fallibility, vulnerability or illness-and felt that they were constantly 'up for judgment' (TP 1), by themselves, colleagues, the wider profession and the general public. There were no apparent differences in how this was described by men and women in this study.

\section{DPs: taking up the patient role}

Given the above, DPs described taking up the patient role as confusing, at times humiliating and challenging to their identity, eg:

You get into all these sorts of confused ideas about who am I and why am I doing this and what is it about...am I a professional, am I a patient and trying to hold all of that together. (DP 1)

They spoke of the importance of being 'open and honest' (DP 3) but also of striving to be, and be seen to

${ }^{\mathrm{i}}$ Refer www.qsinternational.com for further information on the software.

iiThis included one of the researchers. 
be, 'good patients' (DP 2-5). They were concerned about being judged by another doctor, and 'being seen as demanding' (DP 2). Thus, they reported that they often minimised their difficulties and distress:

Giving my history in an ordered way, attending appointments as instructed...I didn't want to be troublesome. (DP 5)

There's wanting to put on a good face type of situation (DP 2)

In line with this, the DPs spoke of their concern for how the interaction was for the TPs. Even when they were in positions of need, DPs were trying to manage their behaviour and take responsibility for their treating doctor. For example, when the DPs shared unsatisfactory experiences they softened their criticism, often taking the blame on themselves. Typical comments included:

And I suspect it was a thoroughly horrible situation for [psychiatrist] as well. (DP 6)

I didn't want to appear too assertive and too knowledgeable and too threatening. (DP 3)

DPs described their professionally acquired knowledge as sometimes unhelpful in the patient role. Some expressed a view that their training had taught them to focus on diagnosis, rather than getting to know the person or hearing their story. They described knowing how to report symptoms as patients, but lacking the capacity to fully tell their stories or articulate their subjective distress. Others reported that they found it hard to bring forward personal knowledge and experience, or that 'overintellectualising' got in the way of effectively engaging in care:

It [training] keeps you away from a feeling state...allows you to stay even more in your head. (DP 4)

You end up talking about brain biochemistry to your doctor which doesn't fix the problem at all (DP 7)

I wasn't listening to the cry of my heart, I wasn't listening to my pain, I wasn't listening to the truth I knew about what had happened and who I was. (DP 3)

\section{Relationship with the TP from the DP perspective}

Forming a therapeutic relationship with a professional peer was difficult for DPs. Indeed, several participants highlighted that they did not find it helpful to engage with their TP as a colleague, but preferred them to give authoritative recommendations. While some described feeling relieved when such recommendations were given, others rejected them. However, they generally did not let the TP know of their differing views, with some reporting that they appeared compliant, but then disengaged from the therapeutic relationship:
[Psychiatrist] immediately made up his/her mind that it was clearly biological cause which I totally disagreed with and that was fine, let him/her talk and ramble on. (DP 7)

I'm actually not going back now, that's me, I'll just get your okay to increase my prescription and say, I'm just going to go now and I'll go to my GP, (DP 6)

For some, a reluctance to voice disagreement or dissatisfaction was because they feared losing the relationship or being pathologised if they criticised their psychiatrist:

Doctors don't want to know if their patients are angry with them. Doctors want to feel really good and helpful and wonderful. (DP 3)

There were exceptions to this. One DP was comfortable openly challenging their psychiatrist, as evident in the following interview extract:

I said I want another option. I have no bipolar disorder...I was very clear about it. (DP 8)

\section{Treatment experiences}

DPs' experiences of being in treatment and being diagnosed as having something 'wrong' with them was challenging - and the research identified a broad spectrum of responses to having their experience conceptualised within a biomedical model. Some found it helpful, some moved between accepting and rejecting it, some railed against it—and others found it unhelpful:

[Psychiatrist] just said 'you're depressed' and started me on [medication]...it was just again that huge sense of relief that I just, I'm unwell and I'm doing something proper about it. (DP 5)

I went because I thought I was having a few problems. And [psychiatrist] told me I was depressed and that I needed antidepressants and I was devastated (DP 4)

While all DPs described being prescribed medication by their psychiatrists at some stage, some initially selfprescribed (often with unhelpful effects). Again, a spectrum of responses emerged. Many experienced only temporary relief accompanied by unpleasant and distressing side effects. Some became increasingly stressed and unnerved by their non-response to medication or, at worst, to the medication's exacerbation of their distress. Some experienced immediate or gradual relief, whereas one found that being off medication altogether in the context of a therapeutic environment brought healing. Examples of comments in relation to medication included:

I just take it and I don't give it much thought really. Except I know they keep me well and I don't stop. I mean I realized very quickly that they worked. (DP 9) 
I didn't want to be a doctor. I didn't want to be a diagnosis. I didn't want to be on medication. I wanted and needed to be me... as I learned to live with myself then I kind of...this illness thing evaporated. (DP 4)

The two people in the room

A minority of DPs reported that they were able to temporarily 'let go' of their doctor identity, and described this as a long and painful process, and a crucial part of healing. In the following extract, one research participant describes the experience of talking openly about the anxiety they experienced in the doctor role:

I'd never talked to anyone about it before in my life. I didn't know you could, thinking back, I just didn't know you could do that... (DP 10)

DPs described how much they appreciated the TP being available to them as a person, and demonstrating interest in their story. They highlighted the value of feeling safe to talk freely, the initially painful but ultimately positive experience of being able to 'leave the white coat at the door' (DP 4), let go of the doctor role and be themselves with their vulnerability, often more readily with a senior clinician:

Not bound by 'I've got to fill in my risk assessment documents and I've got to give you a diagnosis'. It just felt like, actually 'I'm here and I'm listening to you and I'm going to do whatever I need to.' (DP 6)

When TP's engaged in a more personal way it sometimes created anxiety or raised concerns for DPs. For example, when TPs asked about colleagues they knew in common, one (DP 5) felt concerned about adequate attention to confidentiality. Others described feeling uncomfortable when TP's were open about a lack of diagnostic clarity (DP 7) or rang them at home (DP 6).

\section{TP's: providing ordinary care in the context of the medical culture}

A recurring theme for TPs was the desire to provide 'ordinary care' (TP 2-5). They described working to provide the same level of intervention and professional relationship that they would for any other patient. This included screening, treating and charging DPs in a standard manner. TPs described feeling connected to DPs and aware of the risk that collegiality might compromise care.

TPs generally reported that they enjoyed working with DPs, given that they were a resourceful group, with potential for good treatment outcomes. Some TPs spoke of DPs being no different from other educated, highfunctioning patients. They described the experiences they both have, and common identities as doctors as increasing empathy and facilitating rapport. In this way, some viewed the DPs' medical knowledge as helpful, and providing a connection between the two parties. There was also a sense that this could be happening to them, 'there but for the grace of God...' (TP 5, 6). Overall, interviews with TPs showed a sense of their warmth, generosity and goodwill towards doctors presenting with mental health issues, and a strong desire to contribute to their well-being:

I'm a doctor and I care about doctors, yeah, part of it's about treating your own kind, like helping people in your own family. (TP 2)

Despite the intention to provide ordinary care TPs described making themselves more available to DPs and taking extra measures to maintain confidentiality.

\section{Challenges from the TP perspective}

All TPs described challenging aspects of working with DPs, and some experienced particular discomfort or distress if they found engagement with the DP difficult. For example, it was reported that a tendency for DPs to minimise their concerns led to late presentation and created challenges to assessment and care. Moreover, once in treatment, some DPs demonstrated an impression of cooperation' (TP 7) which often turned out to be false. TPs also spoke about a loss of connectedness following disclosure of certain aspects of the DP's personal life (eg, domestic violence).

TPs highlighted that the collegial relationship which 'sits in between' (TP 4) could make interactions during treatment difficult. They were treating a colleague, and did not want to jeopardise the relationship. They described both 'over-reacting and under-reacting' (TP 4) or overcompensating in terms of informed consent, and being slower to push for particular interventions. This was most acute when facing the possibility of compulsory hospitalisation:

I think if $\mathrm{s} /$ he'd been a lawyer or a policeman or a teacher I might have sectioned him/her...s/he did not get best service because of being a doctor I was so reluctant to section him/her. (TP 6)

TPs spoke of heightened self-monitoring or a desire to impress, given that they were being observed by another doctor. Most described feeling that they were vulnerable to criticism by the wider medical community, should adverse events occur (eg, suicide of a DP).

Overall, the experience for the TP appeared to be heightened positively and negatively when the patient is a doctor. Of note, some TPs highlighted that variable behaviour was common among DPs, which meant that treating this patient group could range from very satisfying to extremely challenging:

Ranged from being extremely enjoyable, rewarding, interesting, worthwhile to being one of those things I had to do but wanted to get out of (TP 2)

They're either being good patients or being really difficult and foul and revolting. (TP 6) 
Good patient behaviour was noted by some TPs but the striving of DPs to be seen as good patients was not described by TPs even when asked about it.

\section{What facilitates an effective psychiatrist-patient relationship from the TP perspective?}

A number of issues were highlighted when discussing factors that had facilitated an effective relationship with DPs. For example, TPs talked about how helpful it was to be older and/or more senior than the doctors they were treating, or to have previous experience in treating DPs. Engaging in supervision with colleagues with experience in this area was particularly beneficial.

Some TPs highlighted the value of their own therapy or illness experience. This included, for example, a greater understanding of the challenges involved and the need to take time off work, as well as the significance of no longer having to report to the Medical Council as an impaired professional:

It's brilliant, I really love the fact that I've been depressed....It stinks as an illness but it's a great extra dimension as a psychiatrist. (TP 5)

I feel really comfortable to talk about [taking time off work] because it's part of my experience and I talk about how I push myself to work, even though I know probably that it would be better for me to take some time out...I find that easier really because there's a bit of a connection yeah. (TP 5)

In contrast, another TP described how the increased understanding of the DP's situation could increase their awareness of the complexity of giving appropriate advice:

I should probably have taken time off....But I didn't so I know how hard it is to. That's the other thing-well if I can do it they can do it....There's also the-well I shouldn't have done it and maybe if someone had told me really firmly you shouldn't do it.... (TP 6)

\section{DISCUSSION}

\section{Strengths and limitations}

This study provides rich and experience-based data in an area with a dearth of research. We have not been able to identify any other published research which explored the engagement of doctors as patients in mental health care. The study is 'user-led' research ${ }^{40}$ with the potential benefits of an insider perspective in increased understanding of the significance of the data and the risks of overinvolvement. ${ }^{41}$ The sample includes doctors with experience of severe illness diagnoses who are barely mentioned in the research. The insider perspectives of both researchers as doctors and particularly PR's insider perspective as a patient of a psychiatrist were essential in recruiting.

There is a self-selection bias and doctors who chose to work in psychiatry after the onset of their DP experience are over-represented which may raise concern about the generalisability of the results. But the similarities between their experience and what has been described by doctors accessing general medical care and other people indicates they are not outliers.

\section{General findings}

The results of this study indicate that when a psychiatrist has a doctor as a patient there is risk of adverse effects on the quality of care and even harm. In the process of building a therapeutic relationship the two people in the room are simultaneously negotiating a personperson relationship, a doctor-doctor relationship and a psychiatrist-patient relationship, all within the medical culture.

From the descriptions of the TPs and DPs it appears that aspects of the medical culture, in particular the limited room for fallibility and vulnerability, vigilance for judgement against falling short of high standards and the tendency to value clinical rather than personal knowledge, affect both doctors in the relationship. This has the risk of the problematic aspects of the medical culture working in negative synergy, as described previously. $^{2} 5{ }^{5} 11$ Understanding of the development and impact of the medical culture is emerging. A bullying culture and high expectations during training, as identified in this study, have been documented over many decades ${ }^{42-44}$ and been demonstrated to be difficult to eradicate. ${ }^{45}$ Much of the process of handing on unhelpful values in medicine is implicit ${ }^{46} 47$ and continues postgraduation. ${ }^{48} 49$ Elements of this 'hidden curriculum' can undermine what is taught explicitly in courses covering areas such as communication skills and ethics that are designed to increase doctors' capacity to develop healing relationships. ${ }^{50}$

The challenges TPs described, in terms of feeling particularly connected to, or identifying with, DPs are analogous to challenges in treating family members. Doctors are often prohibited from treating family members and, where there is no alternative, to focus on offering ordinary care. ${ }^{51}$ The TP's in this study described ordinary care as care they would provide to any other patient. In recent years patient-centred care has become the accepted norm for best practice, or ordinary care. ${ }^{13}$ For doctors, being a doctor is a significant part of our identity. ${ }^{311} 2627$ DPs in this study spoke of both the need to be able to be present as a whole person and the need to leave their white coat at the door. Providing patient-centred, ordinary care for doctors needs to take account of the whole person, including the recognition and understanding of the impact of the medical culture on doctors' identity and needs as a person.

The situation with two doctors may be even more complicated than for a doctor treating a family member. There is a fragility to the connectedness within the medical culture. Regard is not unconditional, but dependent on meeting the unrealistically high standards 
described above. As mentioned, bullying and stigmatising are common. ${ }^{8}{ }^{42-44}$ In addition to the high level of scrutiny and judgement doctors have for themselves and each other there is also the shadow of the medical council with the possibility that the DP could lose their ability to work as a doctor.

DPs in this study described also applying the need to meet these high standards by being 'good patients', despite the contradictions between the role of the patient and that of the doctor. Some of the DPs described using their doctor knowledge to take up this 'good patient' role, giving symptoms but not revealing their whole self, engaging with the TP by seeking and accepting treatment within the biomedical model, valuing the psychiatrist's knowledge more than their own. This involves consideration on all sides, not coming into conflict or questioning, carefully monitoring and judging their own behaviour and aware of the monitoring and judging likely to be going on in the other. DPs for whom this works well could be some of the examples the TPs describe as great doctors to have as patients. However, for several this strategy did not work.

The difficulties described by DP's in this study demonstrate many similarities to doctors' illness experiences cited above. However, this study shows a significantly different finding in regard to the spectrum of responses DPs reported when they experienced psychiatrist's treatment within a biomedical model. A similar spectrum also appears to be present in non-doctor patients. Participants in a multicultural study, who identified a biomedical cause for their illness, experienced more satisfaction with their treatment. ${ }^{52}$ On the other hand, authors with lived experience of psychiatric treatment have described it as harmful and even 'spirit breaking. ${ }^{53-55}$ Double $^{56}$ described the biomedical model as denying the patient as a person. Biomedical models do not appear to be helpful in reducing stigma. ${ }^{57} 58$ Concern about the medicalisation of human distress is increasingly evident. ${ }^{5960}$

Few, if any, psychiatrists would advocate a purely biomedical model. Much as patient-centred medicine was embraced in general medicine in response to the dissatisfaction with the biomedical model ${ }^{61}$ psychiatry has embraced the biopsychosocial model. ${ }^{62}$ However, despite the sustained advocacy and teaching of patient-centred medicine, limited evidence on actual practice indicates that medical practice may still be largely focused on the biomedical aspects of medicine ${ }^{48}$ with a decrease in patient-centred strategies. ${ }^{63}$ It appears that psychiatrists are more likely to focus on the biomedical aspects of patient care than issues of interest to patients as individuals. $^{64} 65$

In terms of what helps create positive DP experiences, seniority was described as a desirable attribute, both by the TPs themselves and the DPs in their psychiatrist. It is of interest what features seniority brings with it, and how to accelerate their development. More senior clinicians may have more awareness of medical culture and be more active in choosing what to assimilate. ${ }^{49}$ Findings that empathy decreases over medical school ${ }^{66}$ indicate the urgency of attending to skills in maintaining truly patient-focused care. Effective interventions are available to increase physician empathy ${ }^{67}$ and increase practice of patient-centred medicine. ${ }^{141568}$ Building of these skills needs to be addressed in an ongoing way for psychiatrists and other doctors alongside maintaining knowledge of advances in biomedical research.

The issues identified by the participants in this study may also be important in developing a therapeutic relationship with other patients. Features of the medical culture may be present more widely. Showing vulnerability in terms of health help-seeking can be challenging for many, particularly for men. ${ }^{67}$ High personal standards and perfectionism are not restricted to doctors. ${ }^{69}$ Similarities in response to the biomedical model are discussed above. Reluctance to challenge clinicians may also be common and expected where there is a perceived power imbalance. ${ }^{70}$ The desire to be a good patient, presenting positively without reporting concerns or demanding attention, is common, and described as a barrier to effective healthcare. ${ }^{71-73}$ Medical knowledge is increasingly available to the general public via the internet. TPs have reasons to feel connected to and identify with a range of patients.

This means that strategies to address the issues raised by the DPs may have a significant contribution to care in general. For example, TPs were not aware that the DPs were striving to be good patients. We may be missing this process in our patients generally, despite decades of awareness of 'good patient' dynamics. ${ }^{71}$

\section{Moving forward}

The risks of adverse experiences identified in this study when a doctor engages with a psychiatrist indicate that extra care needs to be taken of the therapeutic relationship. Inviting reflection on the medical culture and the challenges it entails for the DP taking up the patient role may help both doctors manage its effects. ${ }^{49}$ Giving the DP this paper to read may be helpful. Explicitly checking for minimising, and encouraging the DP to tell their whole story, including their lived experience of 'symptoms', may help avoid some of the pitfalls described by participants in this study. Appropriate supervision is clearly needed.

Small ruptures in the therapeutic alliance in psychotherapy which are effectively addressed are associated with better outcomes. ${ }^{74}{ }^{75}$ However, the results of this study indicate that DPs are likely to conceal a rupture from their psychiatrist. Part of the process of taking care of the psychiatrist-patient relationship is to use specific inquiry to identify any small ruptures in the alliance early by: (1) asking how they are experiencing the consultation, for example, 'How well is this conversation working for you?' (2) checking the agenda, for example, 'are we talking about the right things?' and (3) checking 
how the approach (eg biomedical model, being paternalistic/collaborative) is being experienced-'What are you thinking/feeling about this approach?'.

These strategies also have the potential to be useful for other patients. For example, it may be helpful for patients generally to acknowledge the challenges in engaging as a patient of a psychiatrist, to be supported to bring forward lived experience and tell their story, and use specific inquiry to generate feedback as to their experience of the conversation. All this needs to be specifically researched.

Funding The authors have received NZ $\$ 5000$ from the Oakley Mental Health Research Foundation, which is a not-for-profit trust.

Competing interests None declared.

Ethics approval NZ Ministry of Health Northern Region Ethics Committee.

Provenance and peer review Not commissioned; externally peer reviewed.

Data sharing statement The data in this study are very sensitive in terms of confidentiality and so we would not engage in data sharing. Inquiries could be made from the corresponding author regarding specific issues.

Open Access This is an Open Access article distributed in accordance with the Creative Commons Attribution Non Commercial (CC BY-NC 4.0) license, which permits others to distribute, remix, adapt, build upon this work noncommercially, and license their derivative works on different terms, provided the original work is properly cited and the use is non-commercial. See: http:// creativecommons.org/licenses/by-nc/4.0/

\section{REFERENCES}

1. McKevitt C, Morgan M, Dundas R, et al. Sickness absence and 'working through' illness: a comparison of two professional groups. J Public Health Med 1997;19:295-300.

2. Klitzman R. "Post-residency disease" and the medical self: identity, work, and health care among doctors who become patients. Perspect Biol Med 2006;49:542-52.

3. Henderson M, Brooks SK, Del Busso L, et al. Shame! Self-stigmatisation as an obstacle to sick doctors returning to work: a qualitative study. BMJ Open 2012;2:e001776.

4. Flin R, Yule S, McKenzie L, et al. Attitudes to teamwork and safety in the operating theatre. Surgeon $2006 ; 4: 145-51$.

5. Stanton J, Randal P. Doctors accessing mental-health services: an exploratory study. BMJ Open 2010;1:e000017.

6. McKevitt C, Morgan M. Illness doesn't belong to us. J R Soc Med 1997;90:491-5.

7. Kay M, Mitchell G, Clavarino A, et al. Doctors as patients: a systematic review of doctors' health access and the barriers they experience. Br J Gen Pract 2008;58:501-8.

8. Elliot L, Tan J, Norris S. The mental health of doctors: a systematic literature review. Hawthorn, West Victoria: beyondblue: the national depression initiative, 2010.

9. Myers MF, Gabbard GO. The physician as patient: a clinical handbook for mental health professionals. Arlington: American Psychiatric Publising, 2008.

10. Domeyer-Klenske A, Rosenbaum M. When doctor becomes patient: challenges and strategies in caring for physician-patients. Fam Med 2012;44:471-7.

11. Thompson WT, Cupples ME, Sibbett $\mathrm{CH}$, et al. Challenge of culture, conscience, and contract to general practitioners' care of their own health: qualitative study. BMJ 2001;323:728-31.

12. Forsythe $M$, Calnan M, Wall B. Doctors as patients: postal survey examining consultants and general practitioners adherence to guidelines. BMJ 1999;319:605-8.

13. de Haes $\mathrm{H}$. Dilemmas in patient centeredness and shared decision making: a case for vulnerability. Patient Educ Couns 2006;62:291-8.

14. Jensen BF, Gulbrandsen P, Dahl FA, et al. Effectiveness of a short course in clinical communication skills for hospital doctors: results of a crossover randomized controlled trial (ISRCTN22153332). Patient Educ Couns 2011;84:163-9.
15. Maatouk-Burmann B, Ringel N, Spang J, et al. Improving patient-centered communication: results of a randomized controlled trial. Patient Educ Couns 2016;99:117-24.

16. Say R, Murtagh M, Thomson R. Patients' preference for involvement in medical decision making: a narrative review. Patient Educ Couns 2006;60:102-14.

17. Derksen F, Bensing J, Lagro-Janssen A. Effectiveness of empathy in general practice: a systematic review. $\mathrm{Br} J$ Gen Pract 2013;63:76-84.

18. Priebe $\mathrm{S}, \mathrm{McCabe} \mathrm{R}$. Therapeutic relationships in psychiatry: the basis of therapy of therapy in itself? Int Rev Psychiatry 2008;20: 521-6.

19. Zuroff DC, Blatt SJ. The therapeutic relationship in the brief treatment of depression: contributions to clinical improvement and enhanced adaptive capacities. J Consult Clin Psychol 2006;74:130-40.

20. Martin DJ, Garske JP, Davis MK. Relation of the therapeutic alliance with outcome and other variables: a meta-analytic review. J Consult Clin Psychol 2000;68:438-50.

21. McCabe R, Priebe $\mathrm{S}$. The therapeutic relationship in the treatment of severe mental illness: a review of methods and findings. Int J Soc Psychiatry 2004;50:115-28.

22. Johansson $\mathrm{H}$, Eklund M. Patients' opinion on what constitutes good psychiatric care. Scand J Caring Sci 2003;17:339-46.

23. Priebe S, Watts J, Chase M, et al. Processes of disengagement and engagement in assertive outreach patients: qualitative study. $\mathrm{Br} J$ Psychiatry 2005;187:438-43.

24. Jaye $\mathrm{C}$, Wilson $\mathrm{H}$. When general practitioners become patients. Health 2003;7:201-25.

25. Faltholm Y. "Patients, not doctors, get sick": a study of 15 Swedish physicians on long-term sick leave. Int J Qual Stud Health Well-being 2007;2:19-32.

26. McKevitt C, Morgan M. Anomalous patients: the experiences of doctors with an illness. Sociol Health IIIn 1997;19:644-67.

27. Allibone A, Oakes D, Shannon HS. The health and health care of doctors. J R Coll Gen Pract 1981;31:728-34.

28. Ingstad $B$, Christie $M$. Encounters with illness: the perspective of the sick doctor. Anthropol Med 2001;8:201-10.

29. Doust J, Del Mar C. Why do doctors use treatments that do not work?. BMJ 2004;328:1066.

30. Klitzman R. Improving education on doctor-patient relationships and communication: lessons from doctors who become patients. Acad Med 2006;81:447-53.

31. Fox FE, Rodham KJ, Harris MF, et al. Experiencing 'the other side': a study of empathy and empowerment in general practitioners who have been patients. Qual Health Res 2009;19:1580-8.

32. Rosvold EO, Bjertness E. Illness behaviour among Norwegian physicians. Scand J Public Health 2002;30:125-32.

33. Williams AS. Medical practitioners and their families as patients. Australas Psychiatry 2004;12:18-22.

34. Patton MQ. Qualitative research and evaluation methods. Wiley Online Library, 2005.

35. Randal P. Loving relationship is at the root of recovery. In: Leibrich $\mathrm{J}$ ed. A gift of stories: discovering how to deal with mental illness. University of Otago Press/Mental Health Commission, 1999:137-44.

36. Randal P. Subjective experience of spirituality and psychosis. In: Geekie J, Randal P, Lampshire D, et al, eds. Experiencing psychosis: personal and professional perspectives. London \& New York: Routledge, 2011:57-65.

37. Randal P, Geekie J, Lambrecht I, et al. Dissociation, psychosis, and spirituality: whose voices are we hearing? In: Moskowitz A, Schafer I, Dorahy M, eds. Psychosis, trauma and dissociation: emerging perspectives on severe psychopathology. Chichester: Wiley-Blackwell, 2008:333-45.

38. Fossey E, Harvey C, McDermott F, et al. Understanding and evaluating qualitative research. Aust $N Z J$ Psychiatry 2002;36:717-32.

39. Dwyer SC, Buckle JL. The space between: on being an insider-outsider in qualitative research. Int J Qual Methods 2009;8:54-63.

40. Faulkner A, Thomas P. User-led research and evidence based medicine. Br J Psychiatry 2002;180:1-3.

41. Rose D. Service user views and service user research in the Journa of mental health. J Ment Health 2011;20:423-8.

42. Frank E, Carrera JS, Stratton T, et al. Experiences of belittlement and harassment and their correlates among medical students in the United States: longitudinal survey. BMJ 2006;333:682.

43. Silver HK, Glicken AD. Medical student abuse. Incidence, severity, and significance. JAMA 1990;263:527-32.

44. Miedema B, Maclntyre L, Tatemichi S, et al. How the medical culture contributes to coworker-perpetrated harassment and abuse of family physicians. Ann Fam Med 2012;10:111-17. 
45. Fried $\mathrm{JM}$, Vermillion $\mathrm{M}$, Parker $\mathrm{NH}$, et al. Eradicating medical student mistreatment: a longitudinal study of one institution's efforts. Acad Med 2012;87:1191-8.

46. Lempp $\mathrm{H}$, Seale $\mathrm{C}$. The hidden curriculum in undergraduate medical education: qualitative study of medical students' perceptions of teaching. BMJ 2004;329:770-3.

47. Gaufberg EH, Batalden M, Sands R, et al. The hidden curriculum: what can we learn from third-year medical student narrative reflections? Acad Med 2010;85:1709-16.

48. Apker J, Eggly S. Communicating professional identity in medical socialization: considering the ideological discourse of morning report. Qual Health Res 2004;14:411-29.

49. Gordon J, Markham P, Lipworth W, et al. The dual nature of medical enculturation in postgraduate medical training and practice. Med Educ 2012;46:894-902.

50. Lamiani G, Leone D, Meyer EC, et al. How Italian students learn to become physicians: a qualitative study of the hidden curriculum. Med Teach 2011;33:989-96.

51. Council GM. Treating Family Members. 2011 (cited 18 August 2015). http://www.gmc-uk.org/guidance/10247.asp.

52. McCabe R, Priebe S. Explanatory models of illness in schizophrenia: comparison of four ethnic groups. $\mathrm{Br} J$ Psychiatry 2004:185:25-30.

53. Deegan P. Spirit breaking: when helping professions hurt. Humanist Psychol 1990;18:303-13.

54. Leibrich J, ed. A gift of stories: discovering how to deal with mental illness. University of Otago Press/Mental Health Commission, 1999.

55. Geekie J, Randal P, Lampshire D, et al, eds. Experiencing psychosis: personal and professional perspectives. New York: Routledge, 2011.

56. Double D. The limits of psychiatry. BMJ 2002;324:900-4.

57. Angermeyer MC, Holzinger A, Carta MG, et al. Biogenetic explanations and public acceptance of mental illness: systematic review of population studies. Br J Psychiatry 2011;199:367-72.

58. Rusch LC, Kanter JW, Brondino MJ. A comparison of contextual and biomedical models of stigma reduction for depression with a nonclinical undergraduate sample. J Nerv Ment Dis 2009;197:104-10.

59. Smith DT. The diminished resistance to medicalization in psychiatry: psychoanalysis meets the medical model of mental illness. Soc Ment Health 2014;4:75-91.

60. Moncrieff J. The medicalisation of "ups and downs": the marketing of the new bipolar disorder. Transcult Psychiatry 2014;51: 581-98.
61. Mead N, Bower P. Patient-centredness: a conceptual framework and review of the empirical literature. Soc Sci Med 2000;51: 1087-110

62. Engel GL. The clinical application of the biopsychosocial model. Am J Psychiatry 1980;137:535-44.

63. Bensing JM, Tromp F, van Dulmen S, et al. Shifts in doctor-patient communication between 1986 and 2002: a study of videotaped general practice consultations with hypertension patients. BMC Fam Pract 2006;7:62.

64. Ziolkowska J. The objectifying discourse of doctors' questions. Qualitative analysis of psychiatric interviews. Soc Theory Health 2012;10:292-307.

65. McCabe R, Heath C, Burns T, et al. Engagement of patients with psychosis in the consultation: conversation analytic study. BMJ 2002;325:1148-51.

66. Newton BW, Barber L, Clardy J, et al. Is there hardening of the heart during medical school? Acad Med 2008;83:244-9.

67. Kelm Z, Womer J, Walter JK, et al. Interventions to cultivate physician empathy: a systematic review. BMC Med Educ 2014;14:219.

68. Rao JK, Anderson LA, Inui TS, et al. Communication interventions make a difference in conversations between physicians and patients -a systematic review of the evidence. Med Care 2007;45:

340-9.

69. Shafran R, Mansell W. Perfectionism and psychopathology: a review of research and treatment. Clin Psychol Rev 2001;21:879-906.

70. Lee J. Perceived power imbalance and customer dissatisfaction. Serv Ind J 2010;30:1113-37.

71. Taylor SE. Hospital patient behavior-reactance, helplessness, or control. J Soc Issues 1979;35:156-84.

72. Wittink MN, Barg FK, Gallo JJ. Unwritten rules of talking to doctors about depression: integrating qualitative and quantitative methods. Ann Fam Med 2006;4:302-9.

73. Yates $\mathrm{P}$, Edwards $\mathrm{H}$, Nash $\mathrm{R}$, et al. A randomized controlled trial of a nurse-administered educational intervention for improving cancer pain management in ambulatory settings. Patient Educ Couns 2004:53:227-37.

74. Stiles WB, Glick MJ, Osatuke K, et al. Patterns of alliance development and the rupture-repair hypothesis: are productive relationships U-shaped or V-shaped? J Couns Psychol 2004;51:81-92.

75. Castonguay LG, Constantino MJ, Holtforth MG. The working alliance: where are we and where should we go? Psychotherapy 2006;43:271-9. 